\title{
Gender Differences Regarding Novel Biomarkers and Metabolic Risk Factors in Metformin Treated Type 2 Diabetic Patients
}

\author{
Per E. Wändell ${ }^{*}, 1$, Bruna Gigante ${ }^{2}$, Anna Nixon Andreasson ${ }^{1,3}$ and Axel C. Carlsson ${ }^{1,4}$ \\ ${ }^{I}$ Center for Family and Community Medicine, Karolinska Institutet, Huddinge, Sweden \\ ${ }^{2}$ Division of Cardiovascular Epidemiology, Institute of Environmental Medicine, Karolinska Institutet, Stockholm, \\ Sweden \\ ${ }^{3}$ Stress Research Institute, Stockholm University, Stockholm, Sweden \\ ${ }^{4}$ Department of Public Health and Caring Sciences/Section of Geriatrics, Uppsala University, Uppsala, Sweden
}

\begin{abstract}
We aimed to analyze associations between adiponectin, ghrelin and leptin with anthropometric and metabolic markers in men and women with Metformin-treated type 2 diabetes $(n=53)$, recruited from a trial of relaxation therapies. Anthropometrical measures and fasting blood samples were assessed on three occasions: at baseline, and after 10 and 24 weeks: BMI, waist, HbA1c, insulin, glucose, adiponectin, leptin, fasting ghrelin, high sensitivity C-reactive protein (CRP), tumor necrosis factor $\alpha$ (TNF- $\alpha$ ) and interleukin 6 (IL-6). HOMA2ir and HOMA2s were calculated from fasting glucose and insulin, and adiponectin/leptin and adiponectin/HOMA2ir ratios were calculated.

In men, higher leptin and lower adiponectin/leptin ratio correlated with insulin and insulin resistance, and in women lower ghrelin with insulin and insulin resistance. In multivariate linear regression, higher levels of leptin were associated with insulin resistance among men, but not among women. Among women, insulin resistance was associated with lower adiponectin/leptin ratio and ghrelin. Factor analysis showed that leptin in women was closely related to anthropometric variables, but in men both related to anthropometric and inflammatory variables. Gender differences could indicate different pathophysiologic mechanisms of insulin resistance and type 2 diabetes among men and women, where leptin possibly could be a better marker among men, and ghrelin among women.
\end{abstract}

Keywords: Adiponectin, factor analysis, leptin, ghrelin, insulin resistance, diabetes.

\section{INTRODUCTION}

The prevalence of diabetes is increasing worldwide. Diabetes is closely linked to obesity, especially abdominal obesity, insulin resistance and the metabolic syndrome. Currently used prognostic tools, including fasting glucose, $\mathrm{HbA1c}$, and markers of insulin resistance, are being complemented with novel biomarkers. CRP has been shown to be independently associated with risk of cardiovascular events [1]. Several hormones are associated with obesity, i.e. adipokines such as adiponectin, tumor necrosis factor- $\alpha$ (TNF $\alpha$ ), interleukin-6 (IL-6), and leptin, as well as ghrelin, a peptide hormone secreted from the stomach.

Low adiponectin levels are associated with obesity [2], and increased risk of cardiovascular diseases [3], especially coronary atherosclerosis [4]. Low ghrelin levels are associated with insulin resistance [5], and high levels of leptin are associated with risk of coronary heart disease [6]. Less is known regarding the role of these hormones in subjects with diabetes.

Metformin is recognized as the first line pharmacological treatment of type 2 diabetes. However, as metformin was

Address correspondence to this author at the Center for Family and Community Medicine, Karolinska Institutet, Alfred Nobels Allé 12, S-141 83 Huddinge, Sweden; Tel: +46-8-52488727; Fax: +46-8-52488706;

E-mail: per.wandell@ki.se developed long before these novel biomarkers were measureable, the associations in treated subjects have not been studied thoroughly, compared with newer anti-diabetic drugs.

As diabetic and non-diabetic populations, as well as men and women, vary in their susceptibility to risk factors [7], we hypothesize that these differences also will be reflected in the levels of biomarkers and their relative associations with anthropometric and metabolic risk factors, which may be of clinical importance.

The aim of this study was to analyze the associations between adiponectin, ghrelin and leptin with anthropometric and metabolic markers in men and women with metformin treated type 2 diabetes.

\section{MATERIALS AND METHODOLOGY}

\section{Selection of Patients}

The study sample was recruited from four primary health care centers in Stockholm County in a study comparing tactile massage with relaxation exercises in subjects with type 2 diabetes [8]. Patients diagnosed with type 2 diabetes on treatment with metformin were identified from their electronic patient records. Inclusion criteria were 35-75 years of age and a $\mathrm{HbA} 1 \mathrm{c}$ level of $52-73 \mathrm{mmol} / 1$ (i.e. $6-8 \%$ according to Swedish Mono $\mathrm{S}$ standard, which is approximately 1 percentage unit lower than the Diabetes 
Control and Complications Trial, DCCT, standard). To limit possible effects of genetic differences or effects of migration seen in diabetes [9] and cardiovascular disease [10, 11], the health care centers were asked to recruit Swedish-born subjects with Swedish-born parents for the present study. Exclusion criteria were: heart failure or renal failure, and insulin treatment. Examinations were performed at the baseline investigation, at 10 weeks i.e. the end of the intervention, and finally 12 weeks after the completed intervention. Altogether 53 participants (47\% women), age 43-75 years attended the study. Out of these 21 were on lipid-lowering treatment, 12 men and 9 women. One patient had a myocardial infarction during the follow-up. The study received ethical approval by a regional committee at Karolinska Institute. No effect in metabolic control was seen neither with tactile massage nor relaxation in the original study.

\section{Blood Samples and Methods of Analysis}

The following metabolic and inflammatory markers were analyzed from overnight fasting blood samples: plasma glucose, B-HemoglobinA1c (B-HbA1c), serum insulin, serum adiponectin, serum leptin, fasting serum ghrelin, high sensitivity C-reactive protein (CRP), serum tumor necrosis factor $\alpha$ (TNF- $\alpha$, ELISA, R\&D Systems Europe, Ltd) and serum interleukin 6 (IL-6, high sensitivity ELISA, R\&D Systems Europe, Ltd). A certified research laboratory performed the analyses (The Laboratory of Clinical Chemistry at Karolinska University Hospital). The Homeostatic Model Assessment (HOMA) was used to calculate the insulin resistance and sensitivity by the computer model (HOMA2) [12]. HOMA uses values of fasting glucose and fasting insulin. Ratios for adiponectinHOMA2 insulin resistance [13], and adiponectin/leptin [14], were calculated. These ratios have been shown to be associated with insulin resistance and insulin sensitivity.

\section{Anthropometry}

Weight and length were measured, and body mass index (BMI) was calculated [15]. Waist circumference was measured in a standardized way according the recommendation, i.e. at the midway point between the lower rib and the iliac crest in the upright position [16].

\section{Statistical Data Analysis}

Data were analyzed by the STATA statistical software version 11.

The sample size was based on a power calculation on the intervention with tactile massage in comparison to relaxation exercises [8]. Using $\mathrm{HbAlc}$ as the main outcome variable and assuming a difference of $0.5 \%$ units in improvement between the TM and relaxation groups, as the smallest clinically relevant effect, i.e. an improvement of $0.8 \% v s$ $0.3 \%$, respectively, with standard deviation of 0.6 , this would need 23 subjects in each group, with a power $=0.8$ and alpha $=0.05$.

Correlations between new biomarkers and metabolic risk factors were calculated by Spearman's rank sum test. To minimize the risk of mass significance due to multiple testing, all significant findings from the baseline investigation were double-checked at the 10-week and 3- month follow-ups. Each examination was treated as an individual cross-sectional data set in this study. A two-sided $\mathrm{p}$-value of less than 0.05 was regarded significant.

Linear regression models were created, using values from all three occasions, for men and women, with leptin, adiponectin, adiponectin/leptin ratio, ghrelin, CRP, IL-6 and TNF- $\alpha$ as outcome variables, and age, weight, BMI and waist circumference as explanatory variables. Weight, BMI and waist circumference were not included in the same model due to collinearity. Besides, models were created with insulin resistance measured as HOMA2ir as the outcome variable, and all factors mentioned above as explanatory.

Factor analysis was performed for men and women separately, using data from all three occasions. Only Eigenvalues $>1$ were considered, and factor loading $>0.5$. Different rotations were performed to see if there were any consistent patterns, i.e. in Stata Promax, Varimax, Quartimax and Equamax.

\section{RESULTS}

Characteristics of men and women are shown in Table 1. Men showed higher mean weight and waist circumference, a lower leptin value and higher adiponectin/leptin ratio than women.

Table 1. Mean Values of the Factors Among Men and Women with Metformin-Treated Type 2 Diabetes at the Baseline Investigation

\begin{tabular}{|lccc|}
\hline & Men & Women & Difference \\
N= & $\mathbf{2 8}$ & $\mathbf{2 5}$ & p-Value \\
\hline \hline Age, years & $64.1(6.2)$ & $64.5(9.1)$ & 0.84 \\
Weight, kg & $89.4(10.4)$ & $77.2(12.7)$ & $<0.001$ \\
BMI, kg-m ${ }^{2}$ & $28.1(2.9)$ & $28.6(3.7)$ & 0.57 \\
Waist, cm & $104.1(8.1)$ & $97.6(9.5)$ & 0.012 \\
fP-Glucose, mmol/L & $8.9(2.4)$ & $8.4(1.7)$ & 0.41 \\
B-HbA1c, mmol/mol (IFCC) & $59.8(12.8)$ & $57.5(10.2)$ & 0.48 \\
fS-Insulin, pmol/L & $82.1(50.4)$ & $89.7(45.6)$ & 0.57 \\
HOMA2ir & $1.74(1.14)$ & $1.84(0.89)$ & 0.73 \\
HOMA2s & $75.2(34.4)$ & $72.2(45.9)$ & 0.79 \\
S-Adiponectin, mg/L & $5.8(2.7)$ & $6.9(2.7)$ & 0.13 \\
S-Leptin, microg/L & $10.0(5.6)$ & $23.8(10.4)$ & $<0.001$ \\
fS-Ghrelin, ng/L & $802(250)$ & $815(269)$ & 0.86 \\
Adiponectin/HOMA2ir ratio & $4.8(4.2)$ & $5.5(5.0)$ & 0.64 \\
Adiponectin/leptin ratio & $0.78(0.59)$ & $0.37(0.32)$ & 0.0029 \\
P-CRP, mg/L & $4.6(10.6)$ & $3.0(3.0)$ & 0.45 \\
S-TNF $\alpha$, pg/mL & $3.05(3.48)$ & $2.77(3.39)$ & 0.77 \\
S-IL-6, ng/L & $1.47(1.15)$ & $1.64(0.87)$ & 0.55 \\
\hline HOMA2=Homeostasis Model 2, ir=insulin resistance, s=sensitivity. & \\
& & & \\
& & & \\
& & & \\
& & &
\end{tabular}

Correlations between different markers of insulin resistance or sensitivity, and adipokines or markers of inflammation, at baseline are shown for men in Table $\mathbf{2}$ and women in Table 3. For men, the strongest significant correlations (at least $\mathrm{p}<0.01$ ) at all three occasions were 
Table 2. Correlation by Spearman's Test Among Men with Metformin-Treated Type 2 Diabetes (n=28) at Baseline, Time 2 and Time 3

\begin{tabular}{|c|c|c|c|c|c|c|c|}
\hline & Weight & BMI & Waist & Glucose & Insulin & HOMA2ir & HOMA2s \\
\hline \multicolumn{8}{|l|}{ Baseline: } \\
\hline Leptin & 0.35 & $0.39 *$ & $0.44 *$ & 0.04 & $0.41 *$ & $0.40^{*}$ & $-0.42 *$ \\
\hline Ghrelin & -0.12 & -0.24 & 0.07 & 0.04 & -0.29 & -0.30 & 0.29 \\
\hline CRP & 0.33 & $0.47 *$ & 0.37 & 0.01 & 0.35 & 0.35 & $-0.38 *$ \\
\hline $\mathrm{TNF} \alpha$ & -0.19 & -0.10 & -0.27 & -0.03 & -0.07 & -0.09 & 0.08 \\
\hline IL-6 & -0.15 & -0.12 & 0.04 & -0.15 & 0.04 & -0.01 & 0.01 \\
\hline \multicolumn{8}{|l|}{ Time 2: } \\
\hline Ghrelin & -0.06 & -0.24 & -0.15 & -0.03 & -0.38 & -0.26 & 0.27 \\
\hline Adiponectin/HOMA2ir ratio & -0.20 & -0.37 & -0.31 & -0.16 & $-0.65 * * *$ & $-0.63 * *$ & $0.65^{* * *}$ \\
\hline Adiponectin/leptin ratio & -0.36 & $-0.52 * *$ & $-0.51 * *$ & -0.05 & $-0.53 * *$ & $-0.65^{* * *}$ & $0.68^{* * *}$ \\
\hline CRP & 0.27 & $0.46^{*}$ & 0.34 & 0.02 & 0.25 & 0.28 & -0.30 \\
\hline $\mathrm{TNF} \alpha$ & -0.19 & -0.04 & -0.16 & 0.15 & 0.09 & -0.09 & 0.09 \\
\hline IL-6 & 0.13 & 0.30 & 0.22 & -0.18 & 0.03 & 0.05 & -0.09 \\
\hline \multicolumn{8}{|l|}{ Time 3: } \\
\hline Adiponectin & -0.38 & $-0.49 *$ & $-0.48^{*}$ & -0.10 & -0.28 & -0.32 & 0.32 \\
\hline Leptin & $0.71 * * *$ & $0.65^{* *}$ & $0.71 * * *$ & -0.14 & 0.40 & 0.38 & -0.39 \\
\hline IL-6 & -0.07 & 0.09 & 0.02 & -0.30 & 0.17 & 0.12 & -0.12 \\
\hline
\end{tabular}

HOMA2 $=$ Homeostasis Model 2, ir $=$ insulin resistance, $\mathrm{s}=$ sensitivity.

$*$ : $\mathrm{p}<0.05$ but $\geq 0.01, * *$ : $<<0.01$ but $\geq 0.001, * * *: \mathrm{p}<0.001$

found between the adiponectin/HOMA2ir ratio and the following variables: insulin, HOMA2ir and HOMA2s. Significant correlations were also found with a p-level of at least $<0.05$ on all occasions as regards leptin with both BMI and waist; and as regards adiponectin/leptin ratio with the following variables: BMI, insulin, HOMA2ir and HOMA2s. For four of the correlations significant values $(<0.05)$ were found on two out of three occasions, i.e. between leptin and the following variables: insulin, HOMA2ir and HOMA2s; and between CRP and BMI.

For women, the strongest significant correlations (at least $\mathrm{p}<0.01$ ) were found between leptin with weight and BMI; and between adiponectin/HOMA2ir ratio with the following variables: insulin, HOMA2ir and HOMA2s. Correlations were also found with a p-level of at least $<0.05$ on all occasions as regards leptin and waist circumference; between ghrelin with the following variables: insulin and HOMA2ir; and between adiponectin/leptin ratio with the following variables: weight and waist circumference. Besides, values were at least $p<0.05$ on two out of three occasions for correlations between adiponectin and HOMA2ir; ghrelin and HOMA2s; and adiponectin/leptin ratio and BMI.

Results of multivariate linear regression models for men and women separately are shown in Table 4 . Both leptin and adiponectin increased significantly with older age, and for leptin more so among women. Besides, CRP increased in men but decreased in women with older age. Leptin increased in men by waist and in women by weight, adiponectin/leptin ratio decreased by waist in men and by weight in women, and adiponectin and ghrelin decreased by BMI in men and ghrelin by waist in women. Besides, CRP increased by BMI in men. Insulin resistance assessed by HOMA2 decreased in men by adiponectin and increased by leptin, while in women decreased by adiponectin/leptin ratio and ghrelin. In univariate test, leptin was un-significant 
Table 3. Correlations by Spearman's Test Among Women with Metformin-Treated Type 2 Diabetes (n=25) at Baseline

\begin{tabular}{|c|c|c|c|c|c|c|c|}
\hline & Weight & BMI & Waist & Glucose & Insulin & HOMA2ir & HOMA2s \\
\hline \multicolumn{8}{|l|}{ Baseline: } \\
\hline Leptin & $0.70 * * *$ & $0.72 * * *$ & $0.66 * * *$ & -0.21 & 0.05 & 0.02 & -0.01 \\
\hline Ghrelin & -0.10 & -0.24 & -0.36 & $0.44 *$ & $-0.42 *$ & $-0.41^{*}$ & 0.40 \\
\hline CRP & $0.42 *$ & 0.39 & 0.37 & 0.16 & 0.03 & 0.05 & -0.03 \\
\hline $\mathrm{TNF} \alpha$ & 0.04 & 0.04 & -0.13 & 0.20 & 0.02 & 0.02 & -0.05 \\
\hline IL-6 & 0.02 & -0.07 & -0.02 & 0.25 & -0.05 & -0.02 & 0.03 \\
\hline \multicolumn{8}{|l|}{ Time 2: } \\
\hline Ghrelin & -0.11 & -0.17 & -0.18 & 0.42 & $-0.69 * * *$ & $-0.66^{* * *}$ & $0.65 * *$ \\
\hline Adiponectin/HOMA2ir ratio & -0.11 & -0.17 & -0.33 & 0.09 & $-0.96 * * *$ & $0.95^{* * *}$ & $0.95^{* * *}$ \\
\hline Adiponectin/leptin ratio & $-0.43 *$ & 0.37 & $-0.44 *$ & 0.01 & -0.19 & -0.23 & 0.21 \\
\hline CRP & 0.22 & 0.13 & 0.18 & 0.18 & -0.40 & -0.37 & 0.38 \\
\hline $\mathrm{TNF} \alpha$ & -0.34 & -0.29 & -0.41 & 0.27 & -0.29 & -0.27 & 0.24 \\
\hline IL-6 & -0.11 & -0.13 & 0.09 & 0.03 & -0.34 & -0.35 & 0.35 \\
\hline \multicolumn{8}{|l|}{ Time 3: } \\
\hline Adiponectin & -0.27 & -0.16 & -0.24 & 0.05 & -0.34 & -0.31 & 0.32 \\
\hline Leptin & $0.67 * * *$ & $0.71 * * *$ & $0.63 * *$ & -0.10 & 0.19 & 0.25 & -0.24 \\
\hline IL-6 & 0.12 & 0.05 & 0.15 & -0.10 & -0.19 & -0.21 & 0.21 \\
\hline
\end{tabular}

HOMA2 $=$ Homeostasis Model 2 , ir $=$ insulin resistance, $\mathrm{s}=$ sensitivity.

${ }^{*}: \mathrm{p}<0.05$ but $\geq 0.01,{ }^{* *}: \mathrm{p}<0.01$ but $\geq 0.001,{ }^{* * *}: \mathrm{p}<0.001$.

among women and ghrelin among men, while among women adiponectin was associated with decreased insulin resistance.

Factor analysis was performed using data from all three occasions for men and women separately. Among men six factors were identified, with one factor of insulin-related factors (including insulin resistance and sensitivity), one with anthropometric factors (weight, BMI and waist), one with adiponectin-related factors (including the ratios with adiponectin included), one with glucose (and HbAlc when included in the analyses), one inflammatory (with CRP and IL-6), and finally one with TNF- $\alpha$ and ghrelin. Leptin showed similar factor loadings with two of the factors, i.e. the anthropometric and the inflammatory, and was included in the anthropometric factor in three of the rotations, with factor loadings between 0.48 and 0.54 , and in the inflammatory in one rotation (Equamax), with factor loading 0.58 .
For women, 5 factors were identified, with more differences between the rotations than among men. One factor included insulin-related factors, one anthropometric, one glucose-related, one inflammatory (with CRP and IL-6) and the last one TNF- $\alpha$. Adiponectin was included in the inflammatory factor in three of the rotations, with factor loadings at $0.62-0.63$, and in the insulin-related factor in one rotation (Equamax), with factor loading 0.83. Leptin was included in the anthropometric factor, with factor loadings between 0.88 and 0.96 . Ghrelin was included in the insulinrelated factor in three rotations, with factor loadings between 0.61 and 0.68 , and in the TNF- $\alpha$ factor in one rotation (Equamax), with factor loading 0.60. Insulin sensitivity (HOMA2S) was included in the insulin-related factor in three of the rotations, with factor loadings of $0.90-0.95$, but in the inflammatory factor in one (Equamax), with factor loading 0.52 . The adiponectin/HOMA2 ir ratio was included in the insulin-related factor in all rotations, with factor loadings $0.66-0.87$, and the adiponectin/leptin ratio in the 
Table 4. Results of Linear Regression in Metformin-Treated Diabetic Men and Women

\begin{tabular}{|lccc|ccc|}
\hline & \multicolumn{3}{c|}{ Men } & & Women \\
\hline \multicolumn{1}{|c}{ Dependent Factors } & Factor & Coefficient & $\mathbf{9 5 \%}$ CI Interval & Factor & Coefficient & 95\% CI Interval \\
\hline \hline Leptin & Age (per year) & 0.361 & $0.209-0.514$ & Age (per year) & 0.579 & $0.399-0.758$ \\
& Waist (per cm) & 0.416 & $0.292-0.540$ & Weight (per kg) & 0.650 & $0.518-0.782$ \\
Adiponectin & Age (per year) & 0.105 & $0.008-0.202$ & Age (per year) & 0.150 & $0.085-0.215$ \\
& BMI (per unit) & -0.268 & $-0.473--0.064$ & & -0.014 & $-0.020--0.008$ \\
Adiponectin/leptin ratio & Waist (per cm) & -0.047 & $-0.062--0.032$ & Weight (per kg) & -13.18 & $-20.42--5.95$ \\
Ghrelin & BMI (per unit) & -26.40 & $-47.63--5.17$ & Waist (per cm) & -0.136 & $-0.249--0.023$ \\
CRP & Age (per year) & 0.397 & $0.094-0.700$ & Age (per year) & & -0.836 \\
HOMA2ir & BMI (per unit) & 0.994 & $0.352-1.636$ & & $-1.547--0.124$ \\
& Adiponectin & -0.108 & $-0.182--0.035$ & Adiponectin/leptin ratio & Ghrelin & -.0021 \\
\hline
\end{tabular}

As independent factors for leptin, adiponectin, adiponectin/leptin ratio, ghrelin and CRP, age and anthropometric factors were tested, and for HOMA2 insulin resistance (HOMA2ir) also leptin, adiponectin, adiponectin/leptin ratio, ghrelin and CRP, IL-6, and TNF- $\alpha$ were tested, by backward selection. Due to collinearity, weight, BMI and waist were not used in the same model. Beta-coefficients, with $95 \%$ confidence interval, are shown.

anthropometric factor in three rotations, factor loadings 0.58 062, and in the insulin-related factor in one (Equamax), with factor loading 0.63 .

\section{DISCUSSION}

In this sample of metformin-treated individuals with metabolically well controlled type 2 diabetes, we found higher levels of leptin in women than in men as well as different gender patterns for the association between the different variables. Leptin and more so adiponectin/leptin ratio were correlated with metabolic measures among men, while ghrelin showed this correlation pattern among women. Besides, leptin was recognized as a significant factor for insulin resistance among men but not among women.

The factor analysis also showed different patterns among men and women, where leptin clearly groups with the anthropometric factor among women, whereas among men leptin was associated with both the anthropometric and inflammatory factors. Adiponectin, and the ratios including adiponectin, was recognized as a separate factor among men. However, among women adiponectin was included in the inflammatory factor, and also in the insulin-related factor in one rotation, and ghrelin was included in the insulin-related factor in three rotations, and in the TNF- $\alpha$ factor in one rotation. Among men, ghrelin was included in the TNF- $\alpha$ factor in all rotations.

The association between higher levels of leptin and the anthropometric measures, i.e. weight, BMI and waist circumference showed a somewhat stronger and more consistent association in women. As leptin is secreted by adipose tissue and in direct proportion to the amount of body fat, the relation with the anthropometric variables is expected $[17,18]$. The higher level of leptin among women is only partly related to the higher deposit of adipose tissue among women, and it has been hypothesized that women may be resistant to the unfavorable metabolic effects [19]. This is in line with our results of the correlation between leptin and insulin and insulin resistance in men but not in women, and in agreement with findings in an earlier study including obese and never-obese men and women [20]. As regards type 2-diabetes, a review of gender differences concluded, that "men seem more susceptible than women to the consequences of indolence and obesity, possibly due to differences in insulin sensitivity and regional fat deposition" [21]. In Sweden, the prevalence of diabetes in middle age is almost twice as high among men compared with women [22]. One mechanism for this could be that leptin among men but not among women is associated with the development of type 2 diabetes [23, 24], which was contradicted by findings in another study where leptin, irrespective of gender, was associated with worsening of glucose tolerance independently of baseline obesity [25]. Besides, the results in the factor analysis indicated that leptin seems to be a bridge between obesity and inflammation among men. However, when present, diabetes seem to be more deleterious among women than men [26], even if this could be due to the presence of more risk factors among women [27]. In contrast to this, a recent study showed the effects after 5-year follow-up in patients with newly diagnosed patients where an increased mortality was salient in men [28].

In our sample of diabetic patients, adiponectin was associated with lower BMI in linear regression models among men but not in women. In opposite to this, a study on non-diabetic women found that low levels of adiponectin were associated with total and especially central obesity [29]. Besides, recently published findings on a population-based sample showed that adiponectin was correlated with lower amounts of truncal fat but with higher amounts of lower extremity fat [30], indicating that BMI alone seemed to be rather insensitive in relation to circulating adiponectin levels in non-diabetic individuals.

As regard the on adiponectin/leptin ratio, Inoue et al. concluded that it was superior as a marker of insulin resistance than adiponectin or leptin alone among Japanese diabetic patients [14], and our results confirm this for men but not for women. However, we found that the adiponectin/leptin ratio did not show better results than 
leptin alone in relation to the anthropometric measures. The adiponectin-HOMA2 insulin resistance ratio correlated well with the HOMA2ir, which is not surprising, and in agreement with a population-based study on elderly Japanese men and women [13]. As there may be differences in anthropometry, pathophysiology of insulin resistance and body composition it is important to study the effects of these ratios in different population samples.

Ghrelin was associated with lower levels of insulin and HOMA2ir and higher levels in HOMA2s, but only among women, which is not described earlier [5]. One possibility is that the pathophysiological mechanisms in diabetes development differ between men and women, with men more susceptible to leptin produced in the adipose tissue, while in women the protective effects of ghrelin produced in the stomach. At least, our results points to gender differences within this respect.

As regards the levels of leptin and adiponectin in men and women, this is analyzed in an article by Kuo and Halpern [18], using data from 13 studies among men and 15 among women, with in total 4,852 healthy subjects from 43 groups, i.e. 2,416 men and 2,436 women. The subjects in the studies were between 18 and 59 years of age, i.e. younger than in our sample, and with varying BMI, but with mean BMI close to our figures. Mean leptin were 7.75 and 22.5 $\mathrm{ng} / \mathrm{mL}$ (equal to microg/L) among men and women, respectively, i.e. a statistically significant difference, and mean adiponectin 7.31 and $9.31 \mathrm{microg} / \mathrm{mL}$ (equal to $\mathrm{mg} / \mathrm{L}$ ) among men and women, respectively, i.e. non-significant. The mean leptin values were not significantly different from these in our study, but the adiponectin were, for both men and women $(p<0.001)$. As mentioned, our sample consisted of patients with diabetes, treated by metformin, which may explain the lower adiponectin values in our sample. Besides, our sample showed a higher mean age, which also could have affected the values, as both leptin and adiponectin varies by age, as shown in our study.

What also is interesting is the lack of association between IL- 6 and TNF- $\alpha$ to the markers of body fat, metabolic control and insulin resistance, in line with earlier findings among healthy individuals [18], and also confirmed by the factor analysis. IL-6 is secreted in subcutaneous adipose tissue, in contrast to TNF- $\alpha$ [31]. Furthermore, CRP showed a rather weak correlation with anthropometric and metabolic markers, especially among women, which is somewhat surprising. Gender differences have been described, with CRP related to metabolic disturbances among women but not men $[32,33]$. However, the picture in our study could have been obscured by concomitant diseases, why our results on CRP should be interpreted with some caution. Metformin treatment exert some antioxidative properties [34], but it seems uncertain whether this has an effect on our results regarding inflammatory markers. Besides, the correlations between markers of body fat and of insulin resistance were in general rather weak, with the exception of the correlation between waist circumference and HOMA2s (data not shown). In general, non-significant markers may be of interest for prospective studies as they could add information in addition to classical risk factors [35], in contrast to highly correlated factors. However, in a study of diabetic patients
CRP and IL-6 failed to show important contribution to the atherosclerotic process [36].

Some correlations and findings in the factor analysis are quite obvious, and could be seen as confirmation of validity of the analyses and need no further comments. This applies for example the high correlations between the adiponectin/HOMA2 ir ratio, and insulin, HOMA2ir and HOMA2s, as the insulin values is included in all those markers of insulin sensitivity or resistance.

Limitations of this study included a relatively small sample size. To minimize the influence of type II errors, we chose to verify significant findings at the follow-up visits, and to perform additive analyses to the correlation analysis, i.e. linear regression and factor analysis. Our conservative approach where all findings were verified at three timepoints aiming at reducing type I errors may result in type II errors instead. This may be discussed, but we find this approach appealing [7]. Data in the study are cross-sectional, even though measured on three occasions, and no conclusions on causality may be drawn. Thus, the causality of these associations needs to be tested in prospective studies. Besides, we calculated correlations between HOMA2ir and adiponectin/HOMA2ir ratio, as this ratio has been proposed to be valuable, and we are aware of the obvious relationship when HOMA2ir is present in both variables. Furthermore, even estimation of insulin resistance by HOMA2 correlates well with results of euglycemic clamp, it is recommended that results should be interpreted with some caution in small samples as in our study when based only on one measure of insulin [12]. It should also be noted, that $40 \%$ of the patients were prescribed lipidlowering treatment, mostly statins, which also have antiinflammatory effects, why the results of analyses including CRP should be interpreted with caution.

This study has several strengths. The participants were quite homogeneous with fairly strict inclusion criteria, making the results valid for Swedish-born patients with metformin-treated type 2 diabetes. Even if the study design may be regarded as cross-sectional, it is not likely that repeatedly significant findings at follow-ups would be a result of chance. The patients had to most extent no changes made in their therapy, and had ten sessions of relaxation therapy. The fact that the associations remained indicates the findings to be robust and repeatable.

\section{CONCLUSION}

In conclusion, we found gender differences in this sample of metformin-treated diabetic patients, where the insulinrelated factors were correlated with leptin among men, and with ghrelin among women. The clinical significance of the gender differences is unclear, but one possibility is that leptin is linked to insulin resistance among men while women are resistant to the effect to the effect of leptin, and among them other factors are of importance of the development of insulin resistance, e.g. low ghrelin levels. Further studies are needed to illuminate possible gender differences in the pathophysiology of insulin resistance, including both samples with newly diagnosed type 2 diabetes, the metabolic syndrome but without diabetes and non-diabetic subjects. Besides, factors showing no or weak statistical significance to the anthropometric and metabolic 
factors, i.e. CRP, IL- 6 and TNF- $\alpha$, may be of particular interest as prognostic markers in future follow-up studies of type 2 diabetic patients, as they are more likely to add new information and thereby more increment prediction than highly correlated factors.

\section{ACKNOWLEDGEMENTS}

This study was supported by grants from Stockholm County Council. We would also like to thank the attendants of the study, the nurses performing TM and the participating healthcare centers in Stockholm County. Data from Clinical Trial NCT 00960674.

\section{CONFLICT OF INTEREST}

The authors have no conflict of interest to declare.

\section{REFERENCES}

[1] Schulze MB, Rimm EB, Li T, Rifai N, Stampfer MJ, Hu FB. C-reactive protein and incident cardiovascular events among men with diabetes. Diabetes Care 2004; 27: 889-94.

[2] Trujillo ME, Scherer PE. Adiponectin--journey from an adipocyte secretory protein to biomarker of the metabolic syndrome. J Intern Med 2005; $257: 167-75$.

[3] Hui X, Lam KS, Vanhoutte PM, Xu A. Adiponectin and cardiovascular health: an update. Br J Pharmacol 2012; 165: 574-90.

[4] Hasan-Ali H, Abd El-Mottaleb NA, Hamed HB, Abd-Elsayed A. Serum adiponectin and leptin as predictors of the presence and degree of coronary atherosclerosis. Coron Artery Dis 2011;22: 264-9.

[5] Delhanty PJ, van der Lely AJ. Ghrelin and glucose homeostasis. Peptides 2011; 32: 2309-18.

[6] Sattar N, Wannamethee G, Sarwar N, et al. Leptin and coronary heart disease: prospective study and systematic review. J Am Coll Cardiol 2009; 53: 167-75.

[7] Carlsson AC, Nixon Andreasson A, Wandell PE. Poor self-rated health is not associated with a high total allostatic load in type 2 diabetic patients--but high blood pressure is. Diabetes Metab 2011;37: 446-51.

[8] Wandell PE, Carlsson AC, Andersson K, Gafvels C, Tornkvist L. Tactile massage or relaxation exercises do not improve the metabolic control of type 2 diabetics. Open Diabetes J 2010; 3: 6-10.

[9] Wandell PE, Carlsson A, Steiner KH. Prevalence of diabetes among immigrants in the Nordic countries. Curr Diabetes Rev 2010; 6: 126-33.

[10] Carlsson AC, Wandell PE, de Faire U, Hellenius ML. Prevalence of hypertension in immigrants and Swedish-born individuals, a crosssectional study of 60-year-old men and women in Sweden. J Hypertens 2008; 26: 2295-302.

[11] Andersson K, Tornkvist L, Wandell P. Tactile massage within the primary health care setting. Complement Ther Clin Pract 2009; 15: 15860.

[12] Wallace TM, Levy JC, Matthews DR. Use and abuse of HOMA modeling. Diabetes Care 2004; 27: 1487-95.

[13] Nakatochi M, Miyata S, Tanimura D, et al. The ratio of adiponectin to homeostasis model assessment of insulin resistance is a powerful index of each component of metabolic syndrome in an aged Japanese population: results from the KING Study. Diabetes Res Clin Pract 2011; 92: e61-5.

[14] Inoue M, Maehata E, Yano M, Taniyama M, Suzuki S. Correlation between the adiponectin-leptin ratio and parameters of insulin resistance in patients with type 2 diabetes. Metabolism 2005; 54: 281-6.

[15] Wandell PE, Carlsson AC, Theobald H. The association between BMI value and long-term mortality. Int J Obes (Lond) 2009; 33: 577-82.

[16] Lean ME, Han TS, Morrison CE. Waist circumference as a measure for indicating need for weight management. BMJ 1995; 311: 158-61.
[17] Kelesidis T, Kelesidis I, Chou S, Mantzoros CS. Narrative review: the role of leptin in human physiology: emerging clinical applications. Ann Intern Med 2010; 152: 93-100.

[18] Kuo SM, Halpern MM. Lack of association between body mass index and plasma adiponectin levels in healthy adults. Int J Obes (Lond) 2011; 35: 1487-94.

[19] Saad MF, Damani S, Gingerich RL, et al. Sexual dimorphism in plasma leptin concentration. J Clin Endocrinol Metab 1997; 82: 579-84.

[20] Rosenbaum M, Nicolson M, Hirsch J, et al. Effects of gender, body composition, and menopause on plasma concentrations of leptin. J Clin Endocrinol Metab 1996; 81: 3424-7.

[21] Gale EA, Gillespie KM. Diabetes and gender. Diabetologia 2001; 44: 3-15.

[22] Wandell PE, Gafvels C. Patients with type 2 diabetes aged 35-64 years at four primary health care centres in Stockholm County, Sweden. Prevalence and complications in relation to gender and socio-economic status. Diabetes Res Clin Pract 2004; 63: 195-203.

[23] McNeely MJ, Boyko EJ, Weigle DS, et al. Association between baseline plasma leptin levels and subsequent development of diabetes in Japanese Americans. Diabetes Care 1999; 22: 65-70.

[24] Soderberg S, Zimmet P, Tuomilehto J, et al. Leptin predicts the development of diabetes in Mauritian men, but not women: a population-based study. Int J Obes (Lond) 2007; 31: 1126-33.

[25] Franks PW, Brage S, Luan J, et al. Leptin predicts a worsening of the features of the metabolic syndrome independently of obesity. Obes Res 2005; 13: 1476-84.

[26] Natarajan S, Liao Y, Cao G, Lipsitz SR, McGee DL. Sex differences in risk for coronary heart disease mortality associated with diabetes and established coronary heart disease. Arch Intern Med 2003; 163: 173540.

[27] Kanaya AM, Grady D, Barrett-Connor E. Explaining the sex difference in coronary heart disease mortality among patients with type 2 diabetes mellitus: a meta-analysis. Arch Intern Med 2002; 162: 1737-45.

[28] de Fine Olivarius N, Siersma V, Nielsen AB, Hansen LJ, Rosenvinge L, Mogensen CE. Predictors of mortality of patients newly diagnosed with clinical type 2 diabetes: a 5-year follow up study. BMC Endocr Disord 2010; 10: 14.

[29] Gavrila A, Chan JL, Yiannakouris N, et al. Serum adiponectin levels are inversely associated with overall and central fat distribution but are not directly regulated by acute fasting or leptin administration in humans: cross-sectional and interventional studies. J Clin Endocrinol Metab 2003; 88: 4823-31.

[30] Turer AT, Khera A, Ayers CR, et al. Adipose tissue mass and location affect circulating adiponectin levels. Diabetologia 2011; 54: 2515-24.

[31] Mohamed-Ali V, Goodrick S, Rawesh A, et al. Subcutaneous adipose tissue releases interleukin-6, but not tumor necrosis factor-alpha, in vivo. J Clin Endocrinol Metab 1997; 82: 4196-200.

[32] Han TS, Sattar N, Williams K, Gonzalez-Villalpando C, Lean ME, Haffner SM. Prospective study of C-reactive protein in relation to the development of diabetes and metabolic syndrome in the Mexico City Diabetes Study. Diabetes Care 2002; 25: 2016-21.

[33] Rutter MK, Meigs JB, Sullivan LM, D'Agostino RB, Sr., Wilson PW. C-reactive protein, the metabolic syndrome, and prediction of cardiovascular events in the Framingham Offspring Study. Circulation 2004; 110: 380-5.

[34] Chakraborty A, Chowdhury S, Bhattacharyya M. Effect of metformin on oxidative stress, nitrosative stress and inflammatory biomarkers in type 2 diabetes patients. Diabetes Res Clin Pract 2011; 93: 56-62.

[35] Wang TJ. Assessing the role of circulating, genetic, and imaging biomarkers in cardiovascular risk prediction. Circulation 2011; 123: $551-65$.

[36] Anand DV, Lahiri A, Lim E, Hopkins D, Corder R. The relationship between plasma osteoprotegerin levels and coronary artery calcification in uncomplicated type 2 diabetic subjects. J Am Coll Cardiol 2006; 47: $1850-7$. 